\title{
Structure, Rheology, and Electrokinetics of Soft Colloidal Suspension Electrolytes
}

Snehashis Choudhury ${ }^{\mathrm{a}}$, Gaojin Li ${ }^{\mathrm{a}}$, Rohit R. Singh ${ }^{\mathrm{a}}$, Alexander Warren ${ }^{\mathrm{a}}$, Xiaotun Liu ${ }^{\mathrm{a}}$ \& Lynden A. Archer ${ }^{a *}$

${ }^{a}$ School of Chemical and Biomolecular Engineering, Cornell University, Ithaca, NY 14853

Correspondence Email*laa25@cornell.edu 


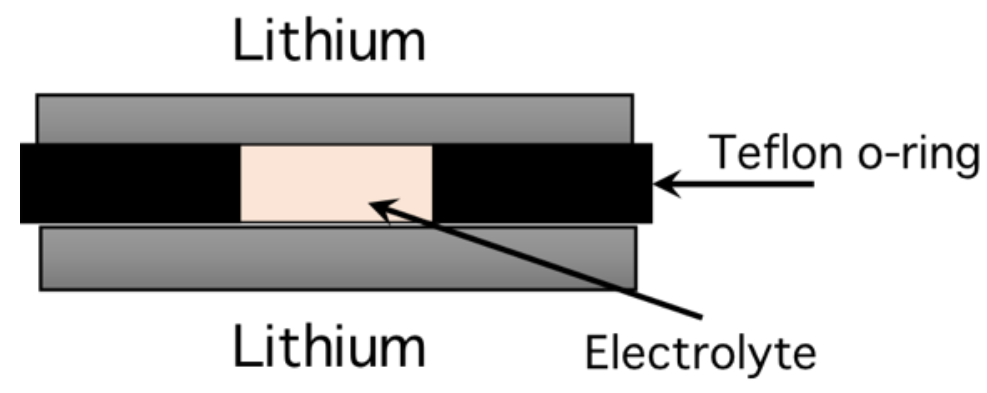

FIG. S1: Electrochemical setup for the current-voltage relationship tests 


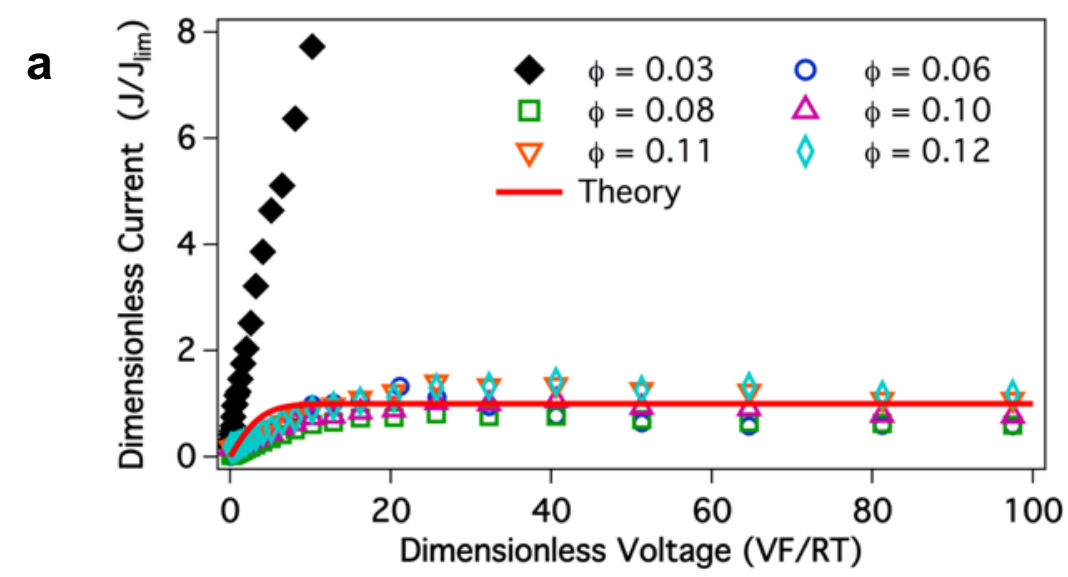

b

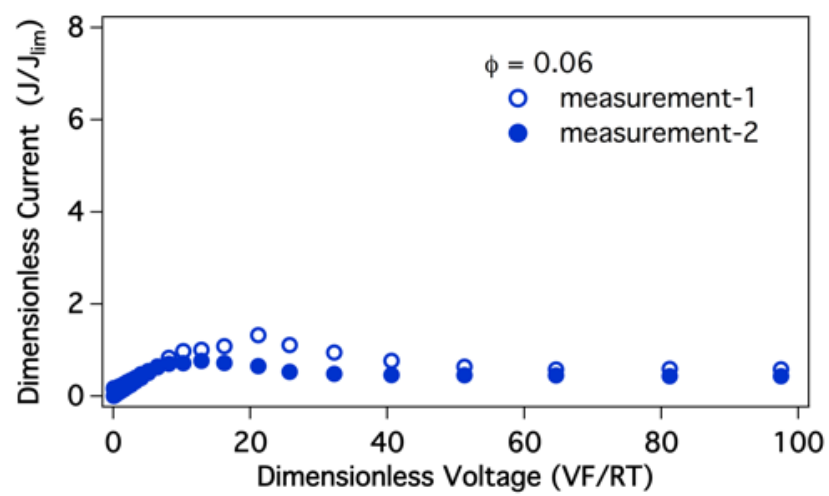

C

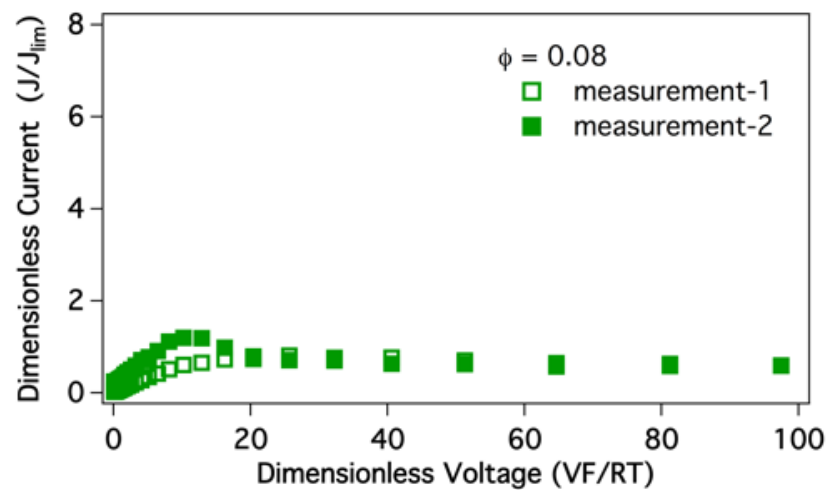

FIG. S2: (a) Dimensionless voltage-current relationship obtained using staircase voltammetry experiment in a symmetric lithium cell with the nanocomposite electrolyte sandwiched using a teflon o-ring. In this measurement, the potentials are held at various values and the steady-state current is obtained. As the voltage scan rate is zero, the diffusion boundary layer thickness is taken as the inter-electrode spacing for calculating the limiting current density. In the figure, the different markers represent various nanocomposite electrolytes while the solid red line is for the prediction for a simple liquid electrolyte using Nernst-Plank model. The experiments were repeated for the electrolytes that indicated similar profiles in (b) and (c). 


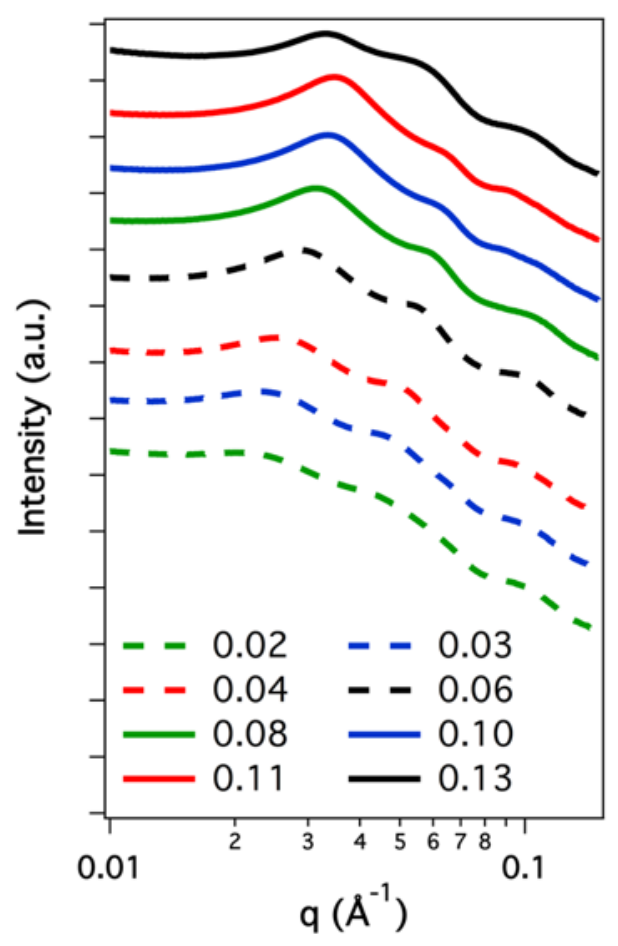

FIG. S3: Scattered intensity obtained using small angle X-ray scattering measurements for various nanocomposite samples plotted as a function of wave vector q. Here the intensity profiles are vertically shifted for clear representation. 

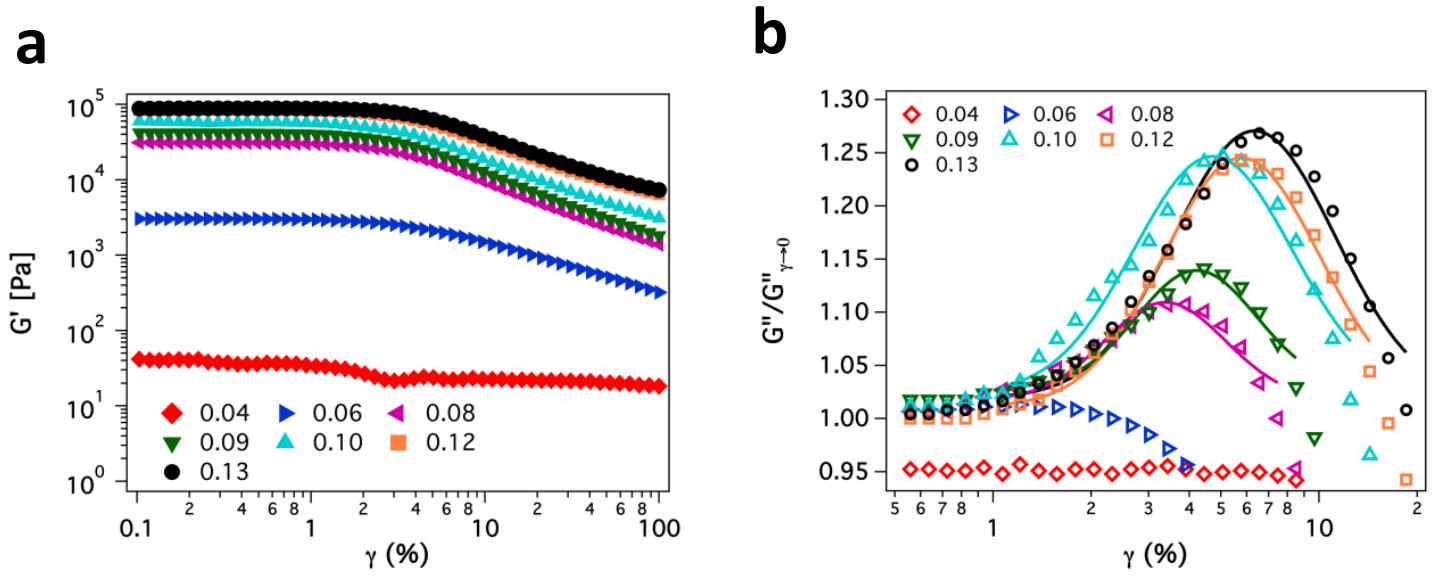

FIG. S4: (a) Storage Modulus and (b) Normalized Loss Modulus obtained from amplitude sweep oscillatory shear measurements at a constant angular frequency of $10 \mathrm{~Hz}$. The legends represents the different core volume fractions. The loss modulus in part $b$ is normalized by the value as strain goes to zero. The lines in part $b$ are log-normal fits to the experimental data. 


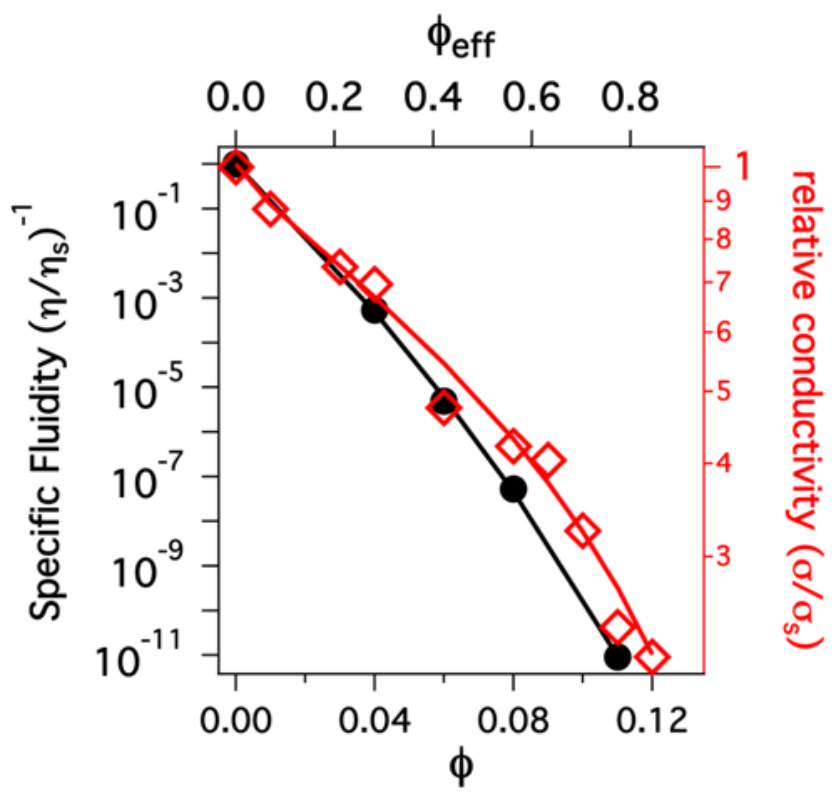

FIG. S5: Variation of the specific fluidity and relative conductivity of the colloidal electrolytes. Both comparisons are done in respect to the values of the particle-free liquid electrolyte. 


\section{Methods}

Experiments

All experiments were performed after an initial equilibration of the suspensions by annealing at $80^{\circ} \mathrm{C}$ overnight under vacuum.

\section{Small Angle X-ray Scattering}

Small angle X-ray scattering (SAXS) measurements were performed at Sector-12-ID-B of Advanced Photon Source (APS) at Argonne National Laboratory using a point collimated X-ray beam. All samples were annealed above the melting temperature for 2 hours before measurements. Samples were smeared on a thermal sample cell, and the measurements were performed at $80^{\circ} \mathrm{C}$. The measured scattering intensity, $(q, \phi)$, depends on wave vector $q$ and particle volume fraction $\varphi$ as $I(q, \phi)=P(q) S(q, \phi)$; where $P(q)$ and $S(q, \phi)$ represent the particle form factor and the interparticle structure factor, respectively.

The particle form factor can be obtained in the limit of infinite dilution given by: $S(q, \phi=0) \approx$ 1 . Thus, we obtained the form factor by performing SAXS measurement of a dilute suspension of $\mathrm{SiO}_{2}$-PEO in water $(\sim 2 \mathrm{wt} . \%)$. The structure factor can then be obtained by normalizing the scattering intensity with the form factor after subtraction of the background.

\section{$\underline{\text { Rheological Measurements }}$}

Oscillatory shear measurements were performed using MCR501 (Anton Paar) rheometer equipped with a $10 \mathrm{~mm}$ cone and a plate fixture at a temperature of $80^{\circ} \mathrm{C}$. All of the suspensions were presheared beyond yielding, to erase any strain history before characterizing their rheological behaviors. Variable-amplitude oscillatory measurements were performed at a fixed angular frequency of $\omega=10 \mathrm{rad} / \mathrm{s}$.

\section{Voltammetry}

The linear scan voltammetry was performed in 2032 coin-cell with two lithium electrodes. The nanocomposite electrolytes were loaded between the two electrodes using a Teflon O-ring spacer (OD: 16mm; ID: $6.35 \mathrm{~mm}$ and thickness: $1 \mathrm{~mm}$ ) that also acts as a separator to prevent any electrical connections. The coin cells were maintained at a fixed temperature of $80^{\circ} \mathrm{C}$ in an environmental chamber and were maintained in a buoyancy-stable position. The measurements were performed by negative voltage scanning using a sweep rate of $1 \mathrm{mV} / \mathrm{s}$.

We also performed potential-hold experiments in similar coin-cells with $\mathrm{Li} / \mathrm{Li}$ symmetric electrode configuration to eliminate the effect of transport related factors. In this method, we imposed a voltage step-ramp protocol and recorded the average current at end of each step. Each voltage step lasted for 5 minutes. 


\section{Theory and Computation}

\section{Brownian Dynamic Simulations}

We consider the motions of spherical colloids in a simple fluid using the Langevin equation with an interparticle force term:

$$
m_{i} \frac{d^{2} r_{i}}{d t^{2}}=-\xi \frac{d r_{i}}{d t}-\sum_{j \neq i} \frac{\partial V_{i j}}{\partial r_{i}}+b_{i}
$$

Here, $m_{i}$ is the mass of particle $\mathrm{i}, r_{i}$ is the position of particle $\mathrm{i}, V_{i j}$ is the interparticle potential between particles $\mathrm{i}$ and $\mathrm{j}, b_{i}$ is a random Brownian force term on particle $\mathrm{i}, \xi$ is a viscous damping coefficient, and $t$ is time. The viscous damping term and the random force term are related in the fluctuation-dissipation theorem:

$$
\left\langle b_{i}(t) b_{j}\left(t^{\prime}\right)\right\rangle=2 k_{B} T \xi \delta_{i j} I \delta\left(t-t^{\prime}\right)
$$

Here, $k_{B}$ is the Boltzmann constant, $\mathrm{T}$ is temperature, and $\boldsymbol{I}$ is the identity tensor. We approximate interparticle interactions using a simple harmonic spring potential:

$$
V_{i j}=\epsilon\left(1-r_{i j} / a_{i j}\right)^{2} \Theta\left(a_{i j}-r_{i j}\right)
$$

Here, $r_{i j}$ is the center-to-center distance between particles $\mathrm{i}$ and $\mathrm{j}, a_{i j}$ is the sum of the radii of particles $\mathrm{i}$ and $\mathrm{j}, a_{i j}=a_{i}+a_{j}, \Theta$ is the Heaviside function, and $\epsilon$ is a constant. We chose $\epsilon=$ $345 k_{B} T$, which is consistent with the value obtained from the relation, $\epsilon=k_{B} T a^{3} /\left(G_{o}\right)$, where $a$ is the radius of polymer-grafted nanoparticle $\sim 10 \mathrm{~nm}$ and $G_{o} \approx 1.8 \mathrm{MPa}$ is the empirical plateau modulus of an entangled $\mathrm{PEO}$ at $80^{\circ} \mathrm{C}$.

The first equation can be integrated with respect to time, and the term on the left side can be neglected in the viscous limit, to give an expression for the time evolution of the positions of the particles. We simulated the motion of a system composed of $N=1000$ colloidal particles in a periodic cubic unit cell at different volume fractions. The simulations were used to determine how the radial distribution function $\mathrm{g}(\mathrm{r})$ changes with particle volume fraction. The diameters of the particles were randomly chosen from a normal distribution with mean $a_{0}$ and standard deviation $0.1 a_{0}$. Lengths were nondimensionalized by $a_{0}$ and times were nondimensionalized by $\tau=$ $\xi a_{0}^{2} / \epsilon$. The simulations were equilibrated for $10,000 \tau$ before particle configurations were used to compute the radial distribution function. After equilibration, particle coordinates were recorded for an additional $1,000 \tau$. The interparticle distances at each time step were used to calculate the radial distribution function. Also, it is important to note that owing to the harmonic potential function, we are able to simulate volume fractions above unity, which would be unfeasible for a hard-sphere suspension. 


\section{Linear Stability Analysis}

Governing equations: The suspension electrolyte is modeled as a porous medium with ions transport inside the solvent inside the pores of the medium. Consider a binary univalent electrolyte flanked between two flat electrode surfaces at $y=0$ and $y=L$. The governing equations for ion concentrations, electric potential and fluid velocity are, respectively, the Nernst-Planck, Poisson's and the Darcy-Brinkman-Stokes equation

$$
\begin{aligned}
& \frac{\partial C^{+}}{\partial t}+\boldsymbol{u} \cdot \nabla C^{+}=D^{+} \nabla^{2} C^{+}+\frac{D^{+} F}{R T} \nabla \cdot\left(C^{+} \nabla \Phi\right) \\
& \frac{\partial C^{-}}{\partial t}+\boldsymbol{u} \cdot \nabla C^{-}=D^{-} \nabla^{2} C^{-}-\frac{D^{-} F}{R T} \nabla \cdot\left(C^{-} \nabla \Phi\right) \\
& \varepsilon \varepsilon_{0} \nabla^{2} \Phi=e\left(C^{-}-C^{+}\right) \\
& -\nabla p-\frac{\mu}{\mathrm{K}} \boldsymbol{u}+\mu \nabla^{2} \boldsymbol{u}+\varepsilon \varepsilon_{0} \nabla^{2} \Phi \nabla \Phi=0, \quad \nabla \cdot \boldsymbol{u}=0
\end{aligned}
$$

where $C^{+}$and $C^{-}$are the concentrations of cation and anion, $\Phi$ is the electric potential; $\boldsymbol{u}$ the solvent velocity; $p$ pressure. $D^{+}$and $D^{-}$are the diffusivities of cation and anion, $R$ is the ideal gas constant, $T$ is tempertature, $F$ is the Faraday's constant, $\varepsilon$ is the relative permittivity of the electrolyte, $\varepsilon_{0}$ is the vacuum permittivity, $e$ is electron charge, $\mu$ is the viscosity of the electrolyte, $\mathrm{K}$ is the permeability of the porous medium. For the soft colloidal suspensions used in the study, $\mathrm{K} \sim h^{2}$, where $h$ is average mesh size of the network formed by nanoparticles. The above equations have several dimensionless parameters: the normalized double layer thickness $\delta=$ $2 \sqrt{\varepsilon \varepsilon_{0} R T / 2 F^{2} C_{0}} / L$, where $C_{0}$ is the average ion concentration and $L$ is the interelectrode distance. The Peclet number $\mathrm{Pe}=U_{0} L / D_{0}$ which defines the strength of coupling between hydrodynamic and electrostatic effects and is mainly affected by the viscosity of the electrolyte, $U_{0}=\varepsilon \varepsilon_{0}(R T / F)^{2} / \mu L$ is a characteristic velocity determined from balancing the characteristic Maxwell stress $\varepsilon \varepsilon_{0}(R T / F L)^{2}$ and the viscous stress $\eta U_{0} / L, D_{0}=2 D^{+} D^{-} /\left(D^{+}+D^{-}\right)$is the average ion diffusivity. The transference number $t_{c}=D^{+} /\left(D^{+}+D^{-}\right)$characterizes the fraction of the total electrical current carried by the cation ions. The Darcy number $\mathrm{Da}=$ $\mathrm{K} / L^{2}$ characterizes the permeability of the porous structure of the colloidal suspension and is the key parameter in this study. In experiments, the parameters are $\mu=10^{-3} \mathrm{~Pa} . \mathrm{s}, L=1 \mathrm{~mm}, D^{+}=$ $10^{-10} \mathrm{~m}^{2} / \mathrm{s}$ and $t_{c}=0.4$ for lithium ion, we choose the dimensional parameters $\mathrm{Pe}=0.35, \delta=$ $10^{-4}$ and Da varies from $10^{-6}$ (overlapped colloidal suspension) to infinity (controlled case).

Boundary Conditions: We only consider the electroconvection in the electrolyte, the boundary conditions at the two electrode surfaces are 


$$
\begin{aligned}
& \left.C^{+}\right|_{y=0, L}=C_{0},\left.\quad\left(\frac{\partial C^{-}}{\partial y}-\frac{F C^{-}}{R T} \frac{\partial \Phi}{\partial y}\right)\right|_{y=0, L}=0 \\
& \left.\boldsymbol{n} \cdot \boldsymbol{u}\right|_{y=0, L}=0,\left.\quad(\mathbf{I}-\boldsymbol{n n}) \cdot \boldsymbol{u}\right|_{y=0, L}=0 \\
& \left.\Phi\right|_{y=0}=0,\left.\quad \Phi\right|_{y=L}=V
\end{aligned}
$$

The first two equations represent a fixed cation surface concentration and a zero anion flux conditions predicated by impermeability of the electrodes. The second equations represent the no slip and no penetration boundary conditions of the electrolyte on both surfaces. Here, $\boldsymbol{n}$ is the normal direction and $\mathbf{I}$ is the identity tensor. The third equation represents the applied voltage $V$ at the cathode.

Analytical Approach: The linear stability analysis is performed on a steady, $x$-independent base state solution $C^{ \pm}(y)$ and $\Phi(y)$, the small perturbations are then added to the base solution as sinusoid waves of wavenumber $k$ and growth rate $\sigma$, i.e., $c^{ \pm}(x, y, t)=c^{ \pm}(y) \mathrm{e}^{\mathrm{i}(k x+\sigma t)}$, $\phi(x, y, t)=\phi(y) \mathrm{e}^{\mathrm{i}(k x+\sigma t)}$ and $v(x, y, t)=v(y) \mathrm{e}^{\mathrm{i}(k x+\sigma t)}$. In the following, we use the capital letters to represent the base solution, and small letters for the perturbed variables. The governing equation for the base state is

$$
\begin{aligned}
& \frac{\partial C^{+}}{\partial y}+\frac{F}{R T} C^{+} \frac{\partial \Phi}{\partial y}=\frac{I}{D^{+}}, \quad \frac{\partial C^{-}}{\partial y}-\frac{F C^{-}}{R T} \frac{\partial \Phi}{\partial y}=0 \\
& \varepsilon \varepsilon_{0} \frac{\partial^{2} \Phi}{\partial y^{2}}=e\left(C^{-}-C^{+}\right)
\end{aligned}
$$

with boundary conditions

$$
\begin{aligned}
& \left.C^{+}\right|_{y=0}=C_{0} \\
& \left.\left(\frac{\partial C^{-}}{\partial y}-\frac{F C^{-}}{R T} \frac{\partial \Phi}{\partial y}\right)\right|_{y=0, L}=0, \quad \int_{0}^{L} C^{-} d y=C_{0} L \\
& \left.\Phi\right|_{y=0}=0,\left.\quad \Phi\right|_{y=L}=V
\end{aligned}
$$

where $I$ is a constant current (depends on $V$ ) that needs to be solved. The second boundary condition for the anion represents that the total anion concentration is conserved in the electrolyted because of the no-flux conditions at both electrode surfaces. The nonlinear equations for the base state are numerically solved using the Newtonian method.

For the perturbed variables, the governing equations are 


$$
\begin{aligned}
& \sigma c^{+}+v \frac{\partial C^{+}}{\partial y}=D^{+}\left[\frac{\partial^{2} c^{+}}{\partial y^{2}}-k^{2} c^{+}-\frac{k^{2} F}{R T} C^{+} \phi+\frac{F}{R T} \frac{\partial}{\partial y}\left(C^{+} \frac{\partial \phi}{\partial y}+c^{+} \frac{\partial \Phi}{\partial y}\right)\right] \\
& \sigma c^{-}+v \frac{\partial C^{-}}{\partial y}=D^{-}\left[\frac{\partial^{2} c^{-}}{\partial y^{2}}-k^{2} c^{+}+\frac{k^{2} F}{R T} C^{-} \phi-\frac{F}{R T} \frac{\partial}{\partial y}\left(C^{-} \frac{\partial \phi}{\partial y}+c^{-} \frac{\partial \Phi}{\partial y}\right)\right] \\
& \varepsilon \varepsilon_{0}\left(\frac{\partial^{2} \phi}{\partial y^{2}}-k^{2} \phi\right)=e\left(c^{-}-c^{+}\right) \\
& \frac{\partial^{4} v}{\partial y^{4}}-\left(2 k^{2}+\frac{1}{\mathrm{~K}}\right) \frac{\partial^{2} v}{\partial y^{2}}+\left(k^{4}+\frac{k^{2}}{\mathrm{~K}}\right) v=\frac{k^{2} \varepsilon \varepsilon_{0}}{\mu}\left[\left(\frac{\partial^{2} \phi}{\partial y^{2}}-k^{2} \phi\right) \frac{\partial \Phi}{\partial y}-\phi \frac{\partial^{3} \Phi}{\partial y^{3}}\right]
\end{aligned}
$$

with boundary conditions

$$
\begin{aligned}
& \left.c^{+}\right|_{y=0, L}=C_{0} \\
& \left.\frac{\partial c^{-}}{\partial y}\right|_{y=0, L}-\left.\frac{F}{R T}\left(C^{-} \frac{\partial \phi}{\partial y}-c^{-} \frac{\partial \Phi}{\partial y}\right)\right|_{y=0, L}=0 \\
& \left.\phi\right|_{y=0, L}=0 \\
& \left.v\right|_{y=0, L}=0,\left.\quad \frac{\partial v}{\partial y}\right|_{y=0, L}=0
\end{aligned}
$$

We then numerically seek the largest growth rate of a perturbation at a given wavenumber using the shooting method. 